\title{
Breaking the Heisenberg limit with inefficient detectors
}

\author{
José Beltrán and Alfredo Luis* \\ Departamento de Óptica, Facultad de Ciencias Físicas, Universidad Complutense, 28040 Madrid, Spain
}

(Received 22 May 2005; published 3 October 2005)

\begin{abstract}
We analyze the performance of nonlinear detection schemes surpassing the Heisenberg limit with semiclassical input states when using realistic detectors having nonunit detection efficiency.

DOI: $10.1103 /$ PhysRevA.72.045801

PACS number(s): 42.50.Dv, 03.65.-w
\end{abstract}

\section{INTRODUCTION}

Quantum fluctuations limit the precision of measurements leading to different quantum limits in different contexts $[1,2]$. The continual improvement of experimental techniques and the interest in performing more accurate measurements compel the thorough examination of quantum limits, their actual range of application, and even the possibility of overcoming them. This effort has led to discover that some quantum limits previously taken for granted are actually not universal, being surpassed by suitable detection schemes [3-5].

In particular, it has been recently shown that nonlinear transformations can break the Heisenberg limit [6]. The Heisenberg limit states that the minimum detectable phase shift is of the order of the inverse of the number of photons. It has been shown that this applies only to linear phase shifts, i.e., when the generator of the transformation encoding the signal in the output field state is the photon number operator. For nonlinear transformations the uncertainty can be below the Heisenberg limit, even when using semiclassical input states. Therefore nonlinear transformations can improve the capabilities of current technology via a more efficient use of the energy resources [7].

In this work we analyze to what extent practical imperfections, such as detection inefficiencies, might deteriorate the performance of nonlinear measuring strategies surpassing the Heisenberg limit. This issue is relevant since it is known that other proposals reaching quantum limits are extremely sensitive to practical imperfections that spoil their performance.

In order to avoid detrimental effects as far as possible we focus on schemes beating the Heisenberg limit using semiclassical input states since this choice favors the overall robustness against imperfections. However, this point should be examined with some care since it is known that nonlinear transformations can convert semiclassical states into nonclassical ones which are extremely fragile against practical disturbances [8].

The detection scheme to be analyzed is outlined in Sec. II. The problem we are addressing is an example of parameter estimation where a proper data analysis is crucial, as demonstrated by previous failed attempts to beat quantum limits [9]. For the sake of completeness and rigor we follow two

*Electronic address: alluis@ fis.ucm.es; URL: http://www.ucm.es/ info/gioq strategies of data analysis. In Sec. III we use a simple propagation of errors while in Sec. IV we use the Fisher information and the Cramér-Rao lower bound. For the sake of comparison in the Appendix we outline an equivalent analysis for linear detection schemes reaching the Heisenberg limit using squeezed states.

\section{DETECTION SCHEME}

The detection scheme is outlined in Fig. 1. We consider a single-mode field with complex amplitude operator $a$. The input state undergoing the nonlinear transformation is a semiclassical coherent state $|\alpha\rangle$ with $a|\alpha\rangle=\alpha|\alpha\rangle$. The nonlinear transformation to be considered is of the Kerr-type, so that the field state after the nonlinear transformation is

$$
|\psi\rangle=e^{i \phi\left(a^{\dagger} a\right)^{2}}|\alpha\rangle .
$$

The purpose of the detection is to infer the value of $\phi$ by performing a suitable measurement on $|\psi\rangle$. From a practical perspective, we must regard $\phi$ as a function of a given physical variable, such as time, length, frequency, temperature, stress, et cetera, that is to be monitored by the detection scheme. For example, nonlinear transformations occur in the propagation of light in nonlinear media, so that $\phi$ depends on the length, density, and temperature of the medium, and the frequency, polarization, and intensity of the light, among other variables.

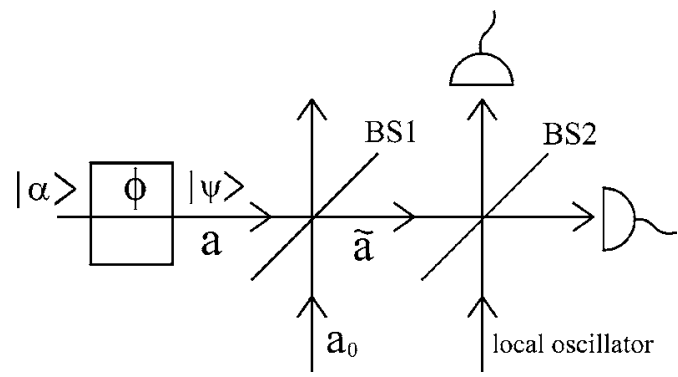

FIG. 1. Outline of the detection scheme showing the input field mode $a$ prepared in a coherent state $|\alpha\rangle$ experiencing a nonlinear unitary transformation encoding the signal $\phi$ in the transformed state $|\psi\rangle$. The beam splitter BS1 describes the effect of nonunit detection efficiencies so that the real scheme performs the homodyne measurement of the quadrature $\tilde{X}$ of the mode $\tilde{a}$ leaving BS1 by mixing the signal beam with a strong local oscillator at beam splitter BS2. 
As a suitable observable we consider the measurement of the quadrature operator $X=\left(a+a^{\dagger}\right) / \sqrt{2}$. This can be achieved in practice by mixing the signal mode at a beam splitter (beam splitter BS2 in Fig. 1) with a strong local oscillator, and detecting the field intensities leaving the two output ports (homodyne detection).

The effect of nonunit detection efficiencies $\eta<1$ can be conveniently described by a fictitious beam splitter of transmittance $\eta$ (BS1 in Fig. 1) placed before an ideal scheme with unit efficiency detectors [10]. Therefore this scheme performs an exact measurement of the quadrature $\tilde{X}=(\tilde{a}$ $\left.+\widetilde{a}^{\dagger}\right) / \sqrt{2}$ of the mode $\tilde{a}=\sqrt{\eta} a+\sqrt{1-\eta} a_{0}$, where $a_{0}$ is a field mode in the vacuum state $|0\rangle_{0}$ mixed with the signal mode $a$ at the beam splitter BS1. In terms of the quadratures of the corresponding modes we have $\tilde{X}=\sqrt{\eta} X+\sqrt{1-\eta} X_{0}$. Since mode $a_{0}$ is in the vacuum state there is a simple relationship between the statistics of $X$ and $\tilde{X}$ (that can be easily derived from characteristic functions) of the form

$$
P_{\eta}(x \mid \phi)=\frac{1}{\sqrt{\pi(1-\eta)}} \int d x^{\prime} e^{-\left(\sqrt{\eta} x^{\prime}-x\right)^{2} /(1-\eta)} P_{1}\left(x^{\prime} \mid \phi\right),
$$

where $P_{\eta}(x \mid \phi)$ is the probability of the outcome $x$ of the measurement of $\tilde{X}$ while $P_{1}(x \mid \phi)$ is the statistics in the ideal case $\eta=1$

$$
P_{1}(x \mid \phi)=\left|\left\langle x\left|e^{i \phi\left(a^{\dagger} a\right)^{2}}\right| \alpha\right\rangle\right|^{2},
$$

$|x\rangle$ being the eigenvector of $X$ with eigenvalue $x$.

\section{PROPAGATION OF UNCERTAINTIES}

The fluctuations $\Delta \tilde{X}$ of the measured observable $\tilde{X}$ imply some uncertainty $\Delta \phi$ for the inferred value of the transformation variable $\phi$. In most cases a very simple data treatment that highlights the fundamental points without involved numerics is available. Since the parameter to be detected is small enough $\phi \ll 1$, which is the case of interest in precision measurements, a linear propagation of uncertainties is adequate leading to [11]

$$
\Delta \phi=\frac{\Delta \tilde{X}}{|d\langle\tilde{X}\rangle / d \phi|}=\frac{\Delta \tilde{X}}{\left|\left\langle\left[\tilde{X},\left(a^{\dagger} a\right)^{2}\right]\right\rangle\right|},
$$

where these magnitudes are evaluated in the state $|\psi\rangle|0\rangle_{0}$ at $\phi=0$ (i.e., in the state $|\alpha\rangle|0\rangle_{0}$ ).

In our case we have

$$
\begin{gathered}
(\Delta \tilde{X})^{2}=\eta(\Delta X)^{2}+(1-\eta)\left(\Delta X_{0}\right)^{2}=\frac{1}{2}, \\
{\left[\tilde{X},\left(a^{\dagger} a\right)^{2}\right]=\sqrt{2 \eta}\left(a^{\dagger} a^{2}-a^{\dagger 2} a\right)+\sqrt{\frac{\eta}{2}}\left(a-a^{\dagger}\right),}
\end{gathered}
$$

leading to $(\Delta \phi)^{2}=1 /\left(8 \eta n^{2} \bar{y}^{2}\right)$, where $\bar{y}=i\left(\alpha^{*}-\alpha\right) / \sqrt{2}$ and $n$ is the mean number of photons $n=|\alpha|^{2}$. For fixed $n$ the minimum $\Delta \phi$ is obtained for maximum $\bar{y}$, so that $\alpha=i \sqrt{n}, \bar{y}$ $=\sqrt{2 n}$, leading to

$$
(\Delta \phi)^{2}=\frac{1}{16 \eta n^{3}} .
$$

We can appreciate that the consequence of nonideal detection is only the reduction of the effective number of photons. This does not deteriorate the overall performance since we can see that even with inefficient detection $(\Delta \phi)^{2}$ still scales as $1 / n^{3}$, which is much better than the Heisenberg limit $1 / n^{2}$. Therefore this kind of detection is robust against practical imperfections.

In general, the physical agent to be detected will give rise also to a linear transformation since the linear and nonlinear susceptibility depend essentially on the same set of variables, so that $\left(a^{\dagger} a\right)^{2}$ should be replaced throughout by a linear combination of $a^{\dagger} a$ and $\left(a^{\dagger} a\right)^{2}$. The relevant point is that the linear and nonlinear contributions lead to a competition of terms in the denominator of Eq. (3.1) with different dependence on the photon number, $n^{1 / 2}$ and $n^{3 / 2}$, respectively. Therefore in order to surpass the Heisenberg limit it is crucial that the nonlinear term should be larger than the linear one. This can occur in the context of electromagnetically induced transparency [12] where giant nonlinear effects arising even at low photon levels allow the predominance of the nonlinear part for large intensities. Moreover, this is actually accompanied by a suppression of the linear susceptibility as shown in Ref. [13]. For other not so optimal implementations the deteriorating effects of the linear part might be avoided by inserting another medium in the path of the local oscillator with a similar linear susceptibility but vanishing nonlinear effects so that the linear parts will mutually compensate (an analog of the compensating plate in Michelson interferometers).

It can be noticed that the nonlinear transformation (2.1) formally coincides with the effect caused by radiation pressure in high power interferometers with mobile mirrors, as it is the case of the detection of gravitational waves $[4,5,14]$. The equivalence suggests that radiation pressure provides another practical implementation of the approach presented in this work. In this regard it must be noted that previous analysis of interferometers with radiation pressure focus on measuring arrangements monitoring the position of the mirrors. Since for collimated beams radiation pressure does not depend on this variable the associated nonlinear transformation does not contribute to the generator of the transformation appearing in the denominator of Eq. (3.1). Instead, it affects the detected observable in the numerator, essentially causing an intensity-dependent rotation of the field quadratures. This alteration can be nevertheless compensated by a suitable choice of the input fields [5], so that the ultimate precision is the same for interferometers with mobile and fixed mirrors [14].

\section{ACCURACY VIA FISHER INFORMATION}

The simple error propagation carried out above might be criticized as being too simple and more adequate to a classical framework than to a quantum one [15]. In this regard the Cramér-Rao lower bound provides a more rigorous approach to statistical parameter estimation in terms of quantum probability distributions [16]. 
The Cramér-Rao lower bound states that the root mean square error $\Delta \theta$ of any unbiased estimator $\theta$ of $\phi$ satisfies the inequality $\Delta \theta \geqslant 1 / \sqrt{F}$, where $F$ is the Fisher information

$$
F(\phi)=\int d x \frac{1}{P_{\eta}(x \mid \phi)}, \quad\left(\frac{\partial P_{\eta}(x \mid \phi)}{\partial \phi}\right)^{2} .
$$

In the following we will consider very small $\phi$ so that the Fisher information is evaluated at the origin $\phi=0$. We also assume the optimum value for the coherent amplitude $\alpha$ $=i \sqrt{n}$ derived above.

For the evaluation of the $\phi$ derivative of $P_{\eta}(x \mid \phi)$ we first compute

$$
\begin{aligned}
\left.\frac{\partial}{\partial \phi}\left\langle x\left|e^{i \phi\left(a^{\dagger} a\right)^{2}}\right| \alpha\right\rangle\right|_{\phi=0} & =i\left\langle x\left|\left(a^{\dagger} a\right)^{2}\right| \alpha\right\rangle \simeq i \alpha^{2}\left\langle x\left|a^{\dagger 2}\right| \alpha\right\rangle \\
& =-i \frac{n}{2}\left(x-\frac{\partial}{\partial x}\right)^{2}\langle x \mid \alpha\rangle \\
& \simeq\left(i n^{2}-\sqrt{8 n^{3}} x\right)\langle x \mid \alpha\rangle,
\end{aligned}
$$

where we have retained only the leading terms in $n$, and we have used $X$-quadrature representation for $X$ and its conjugate operator $Y=i\left(a^{\dagger}-a\right) / \sqrt{2}$. Then we get

$$
P_{1}(x \mid 0)=\frac{1}{\sqrt{\pi}} e^{-x^{2}},\left.\quad \frac{\partial}{\partial \phi} P_{1}(x \mid \phi)\right|_{\phi=0}=-\sqrt{\frac{32 n^{3}}{\pi}} x e^{-x^{2}} .
$$

Using relation (2.2) we finally get

$$
P_{\eta}(x \mid 0)=\frac{1}{\sqrt{\pi}} e^{-x^{2}},\left.\quad \frac{\partial P_{\eta}(x \mid \phi)}{\partial \phi}\right|_{\phi=0}=-\sqrt{\frac{32 \eta n^{3}}{\pi}} x e^{-x^{2}},
$$

leading to

$$
F=16 \eta n^{3}, \quad(\Delta \theta)^{2} \geqslant \frac{1}{16 \eta n^{3}} .
$$

This result confirms the conclusions of the simpler approach in Sec. III. Nonunit quantum efficiency deteriorates the resolution of nonlinear detection schemes very mildly, in sharp contrast to what occurs in linear schemes using nonclassical input states, as recalled in the Appendix . The equality between Eqs. (3.3) and (4.5) can be ascribed to the fact that from Eq. (4.4) and for $\phi \ll 1$ the statistics of the measurement of $\tilde{X}$ can be expressed as a Gaussian

$$
P_{\eta}(x \mid \phi) \simeq \frac{1}{\sqrt{\pi}} e^{-\left(x+\sqrt{8 \eta n^{3}} \phi\right)^{2}} .
$$

\section{CONCLUSIONS}

We have analyzed the resolution of a nonlinear measuring scheme for precision detection with semiclassical input states when we take into account nonunit detection efficiencies. We have shown that the detection scheme is robust against practical imperfections so that the beating of the Heisenberg limit persists even under inefficient detection.
In this regard it is worth noting that there is an extensive literature focusing on the use of nonlinear transformations in order to generate the optimum states for the detection of linear phase shifts $[2,6]$. The scheme analyzed in this work changes the perspective by proposing that the nonlinear transformations should be used to encode the signal to be detected. This measuring strategy would greatly improve the precision and robustness of detection schemes.

\section{ACKNOWLEDGMENT}

A.L. thanks Professor J. Dunningham for valuable comments.

\section{APPENDIX: NONUNIT QUANTUM EFFICIENCY IN LINEAR SCHEMES}

For the sake of comparison and completeness we examine the effect of inefficient detectors in measuring schemes based on linear phase shifts. The basis is the same as illustrated in Fig. 1. There are only two differences: (i) The phase shift is generated by the number operator $a^{\dagger} a$ instead of its square, and (ii) the input state is a coherent squeezed state $|\xi\rangle$, so that $|\psi\rangle=\exp \left(i \phi a^{\dagger} a\right)|\xi\rangle$, where for the state $|\xi\rangle$ we have $\Delta Y$ $=1 / \sqrt{2 \lambda}$, and $\Delta X=\sqrt{\lambda / 2}, \lambda$ being a constant.

The linearized analysis of Sec. III leads to

$$
(\Delta \widetilde{X})^{2}=\frac{\eta \lambda-\eta+1}{2}\left[\tilde{X}, a^{\dagger} a\right]=i \sqrt{\eta} Y,
$$

so that the analog of Eq. (3.1) gives

$$
(\Delta \phi)^{2}=\frac{\eta \lambda-\eta+1}{2 \eta \bar{y}^{2}},
$$

where $\bar{y}=\langle Y\rangle$. Next we consider the optimum resolution for fixed mean number of photons $n=\left\langle a^{\dagger} a\right\rangle$ using the relation

$$
n=\frac{1}{2}\left[\langle X\rangle^{2}+\langle Y\rangle^{2}+(\Delta X)^{2}+(\Delta Y)^{2}-1\right] \simeq \frac{\bar{x}^{2}+\bar{y}^{2}}{2}+\frac{1}{4 \lambda},
$$

where $\bar{x}=\langle X\rangle$ and we have retained just the leading terms in $1 / \lambda$ since after Eq. (3.1) the optimum results will be obtained when $\lambda \ll 1$ so that $\Delta \tilde{X}$ is as small as possible. Then we have

$$
(\Delta \phi)^{2}=\frac{\lambda-\lambda \eta+\lambda^{2} \eta}{\eta\left(4 n \lambda-1-\lambda \bar{x}^{2}\right)} .
$$

For ideal detectors $\eta=1$ the minimum of $\Delta \phi$ for fixed $n$ is obtained for $\lambda=1 /(2 n)$ and $\bar{x}=0$, leading to the Heisenberg limit $(\Delta \phi)^{2}=1 /\left(4 n^{2}\right)$. On the other hand when $\eta<1$ and $n$ $\gg 1$ the minimum $\Delta \phi$ for fixed $n$ is obtained when $\lambda$ $\simeq \sqrt{(1-\eta) /(4 \eta n)}, \bar{x}=0$, and $\bar{y}^{2} \simeq 2 n$, leading to $(\Delta \phi)^{2} \simeq(1$ $-\eta) /(4 \eta n)=\lambda^{2}$. We can appreciate that for nonideal detectors the resolution is far from the Heisenberg limit. As a matter of fact the use of squeezed states reports no benefit, since the same scaling of $\Delta \phi$ with $n$ can be achieved using exclusively semiclassical coherent states. 
On the other hand, in order to compute the Fisher information we assume the optimum input condition $\bar{x}=0$ derived above so that $|\xi\rangle$ is defined by the eigenvalue equation $(X$ $+i \lambda Y)|\xi\rangle=i \lambda \bar{y}|\xi\rangle$, and

$$
\langle x \mid \xi\rangle=\frac{1}{(\pi \lambda)^{1 / 4}} e^{i \bar{y} x} e^{-x^{2} /(2 \lambda)} .
$$

Then we have

$$
\begin{aligned}
\left.\frac{\partial}{\partial \phi}\left\langle x\left|e^{i \phi a^{\dagger} a}\right| \xi\right\rangle\right|_{\phi=0} & =i\left\langle x\left|a^{\dagger} a\right| \xi\right\rangle=\frac{i}{2}\left(x^{2}-\frac{\partial^{2}}{\partial x^{2}}-1\right)\langle x \mid \xi\rangle \\
& \simeq\left[\frac{i}{2}\left(\frac{1}{\lambda}+\bar{y}^{2}\right)-\frac{\bar{y}}{\lambda} x\right]\langle x \mid \xi\rangle,
\end{aligned}
$$

where we have retained the leading terms in $\bar{y}$ and $1 / \lambda$. Finally we get

$$
P_{1}(x \mid 0)=\frac{1}{\sqrt{\pi \lambda}} e^{-x^{2} / \lambda},\left.\quad \frac{\partial P_{1}(x \mid \phi)}{\partial \phi}\right|_{\phi=0}=-\frac{2 \bar{y} x}{\sqrt{\pi \lambda^{3}}} e^{-x^{2} / \lambda}
$$

and

$$
P_{\eta}(x \mid 0)=\frac{1}{\sqrt{\pi(1+\lambda \eta-\eta)}} e^{-x^{2} /(1+\lambda \eta-\eta)}
$$

$$
\left.\frac{\partial P_{\eta}(x \mid \phi)}{\partial \phi}\right|_{\phi=0}=-\frac{2 \bar{y} \sqrt{\eta} x}{\sqrt{\pi(1+\lambda \eta-\eta)^{3}}} e^{-x^{2} /(1+\lambda \eta-\eta)},
$$

leading to

$$
F=\frac{2 \eta \bar{y}^{2}}{\eta \lambda-\eta+1}, \quad(\Delta \theta)^{2} \geqslant \frac{\eta \lambda-\eta+1}{2 \eta \bar{y}^{2}},
$$

which is exactly the same result in Eq. (A2) obtained via error propagation.
[1] C. M. Caves, Phys. Rev. Lett. 45, 75 (1980); W. M. Itano, J. C. Bergquist, J. J. Bollinger, J. M. Gilligan, D. J. Heinzen, F. L. Moore, M. G. Raizen, and D. J. Wineland, Phys. Rev. A 47, 3554 (1993); D. J. Wineland, J. J. Bollinger, W. M. Itano, and D. J. Heinzen, ibid. 50, 67 (1994); Z. Y. Ou, ibid. 55, 2598 (1997); C. Brif, Phys. Lett. A 263, 15 (1999).

[2] A. Luis and L. L. Sánchez-Soto, Progress in Optics, edited by E. Wolf (Elsevier, Amsterdam, 2000), Vol. 41, p. 421, and references therein.

[3] M. Xiao, L.-A. Wu, and H. J. Kimble, Phys. Rev. Lett. 59, 278 (1987); P. Grangier, R. E. Slusher, B. Yurke, and A. LaPorta, ibid. 59, 2153 (1987); E. S. Polzik, J. Carri, and H. J. Kimble, ibid. 68, 3020 (1992); J. L. Sørensen, J. Hald, and E. S. Polzik, ibid. 80, 3487 (1998); H.-A. Bachor, A Guide to Experiments in Quantum Optics (Wiley-VCH, Weinheim, 1998); N. Treps, U. Andersen, B. Buchler, P. K. Lam, A. Maître, H.-A. Bachor, and C. Fabre, Phys. Rev. Lett. 88, 203601 (2002); R. Schnabel, W. P. Bowen, N. Treps, B. Buchler, T. C. Ralph, P. K. Lam, and H.-A. Bachor, Opt. Spectrosc. 94, 651 (2003).

[4] C. M. Caves, Phys. Rev. D 23, 1693 (1981); R. Loudon, Phys. Rev. Lett. 47, 815 (1981); R. S. Bondurant, Phys. Rev. A 34, 3927 (1986); W.-T. Ni, Phys. Rev. D 35, 3002 (1987); A. Luis and L. L. Sánchez-Soto, J. Mod. Opt. 38, 971 (1991); Phys. Rev. A 45, 8228 (1992); A. F. Pace, M. J. Collett, and D. F. Walls, ibid. 47, 3173 (1993).

[5] M. T. Jaekel and S. Reynaud, Europhys. Lett. 13, 301 (1990); A. Luis and L. L. Sánchez-Soto, Opt. Commun. 89, 140 (1992).
[6] A. Luis, Phys. Lett. A 329, 8 (2004).

[7] A. Luis, Phys. Rev. A 65, 025802 (2002); 69, 044101 (2004); J. Opt. B: Quantum Semiclassical Opt. 6, 1 (2004).

[8] G. J. Milburn, Phys. Rev. A 33, 674 (1986); B. Yurke and D. Stoler, Phys. Rev. Lett. 57, 13 (1986); B. C. Sanders, Phys. Rev. A 45, 6811 (1992); B. C. Sanders and D. A. Rice, ibid. 61, 013805 (2000); C. C. Gerry, A. Benmoussa, and R. A. Campos, ibid. 66, 013804 (2002); C. C. Gerry and R. A. Campos, ibid. 68, 025602 (2003).

[9] Z. Hradil and J. Shapiro, Quantum Opt. 4, 31 (1992).

[10] H. P. Yuen and J. H. Shapiro, IEEE Trans. Inf. Theory IT-26, 78 (1980); B. Yurke, Phys. Rev. A 32, 311 (1985); U. Leonhardt and H. Paul, ibid. 48, 4598 (1993); T. Kim, Y. Ha, J. Shin, H. Kim, G. Park, K. Kim, T. G. Noh, and Ch. K. Hong, ibid. 60, 708 (1999).

[11] M. Hillery and L. Mlodinow, Phys. Rev. A 48, 1548 (1993); M. Zawisky, Y. Hasegawa, H. Rauch, Z. Hradil, R. Myska, and J. Peřina, J. Phys. A 31, 551 (1998).

[12] H. Schmidt and A. Imamoğlu, Opt. Lett. 21, 1936 (1996); S. E. Harris and L. V. Hau, Phys. Rev. Lett. 82, 4611 (1999).

[13] S. E. Harris, J. E. Field, and A. Imamoğlu, Phys. Rev. Lett. 64, 1107 (1990); H. Kang and Y. Zhu, ibid. 91, 093601 (2003).

[14] A. Luis, Phys. Rev. A 69, 045801 (2004).

[15] Z. Hradil and J. Řeháček, Phys. Lett. A 334, 267 (2005).

[16] S. L. Braunstein, Phys. Rev. Lett. 69, 3598 (1992); B. R. Frieden, Phys. Lett. A 169, 123 (1992); Physics from Fisher Information: A Unification (Cambridge University Press, Cambridge, England, 1998). 